\section{AB0410 THE INTERPLAY BETWEEN C3 AND C4 AND DISEASE ACTIVITY IN PATIENTS AFFECTED BY RHEUMATOID ARTHRITIS DURING TREATMENT WITH ANTI-IL6 RECEPTOR}

P. Conigliaro $^{1}$, P. Triggianese ${ }^{1}$, M.S. Chimenti ${ }^{1}$, I. Duca ${ }^{2}$, B. Kroegler ${ }^{1}$, R. Perricone ${ }^{1}$. ${ }^{1}$ Rheumatology, Allergology and Clinical Immunology, University of Rome Tor Vergata; ${ }^{2}$ Dipartimento di Medicina Interna e Specialità Mediche, Reumatologia, Sapienza Università di Roma, Rome, Italy

Background: The complement system (CS) is involved in the pathogenesis of rheumatoid arthritis (RA). Evidence reported that high C3 levels C3 in RA patients might reflect a pro-inflammatory status and represent a negative prognostic factor for TNF-inhibitors therapy.

Objectives: We evaluated CS in a cohort of RA patients treated with tocilizumab (TCZ) to explore whether there was any correlation between CS and both disease activity and response to therapy.

Methods: Consecutive outpatients affected by RA who started TCZ treatment i.v. at the Rheumatology Unit of "Policlinico Tor Vergata" in Rome (Italy) were enrolled $(n=25)$ (Table I). We included 25 healthy subjects as controls. Disease activity was assessed by using DAS28 ESR-based while clinical response to therapy by EULAR response criteria. Peripheral blood samples were obtained from all patients at the time of each infusion. Laboratory assessment included ESR, CRP, RF, ACPA, C3, C4, CH50 assay. Clinical and laboratory data were registered at baseline (T0), after 1 (T1), 3 (T3), 6 (T6), 9 (T9), and 12 (T12) months from the beginning of the TCZ treatment.

Results: At baseline, both $\mathrm{C} 3$ and $\mathrm{C} 4$ were significantly higher in RA than in controls (Table 1).

Table 1

\begin{tabular}{|c|c|c|c|}
\hline & $\mathrm{RA}(\mathrm{N}=25)$ & Controls $(\mathrm{N}=25)$ & $P$ value \\
\hline Female (N/\%) & 23/92 & 23/92 & N.S. \\
\hline Age (years) & $65.4 \pm 11.5$ & $60.1 \pm 9.3$ & N.S. \\
\hline Disease duration (years) & $13 \pm 8.2$ & N.A. & N.A. \\
\hline DAS28 & $6.5 \pm 1.3$ & N.A. & N.A. \\
\hline $\mathrm{HAQ}$ & $1.9 \pm 0.8$ & N.A. & N.A. \\
\hline Positivity of RF (N/\%) & $17 / 68$ & N.A. & N.A. \\
\hline Positivity of ACPA (N/\%) & $20 / 80$ & N.A. & N.A. \\
\hline $\operatorname{ESR}(\mathrm{mm} / \mathrm{h})$ & $58 \pm 28$ & N.A. & N.A. \\
\hline $\operatorname{CRP}(\mathrm{mg} / \mathrm{l})$ & $8.5 \pm 7.6$ & N.A. & N.A. \\
\hline C3 (mg/dl) & $119.4 \pm 22.4$ & $104 \pm 18$ & 0.04 \\
\hline $\mathrm{C} 4$ (mg/dll) & $26 \pm 6.7$ & $22 \pm 4$ & 0.013 \\
\hline $\mathrm{CH} 50(\%)$ & $108 \pm 24$ & $100 \pm 18$ & N.S. \\
\hline Therapy: & & N.A. & \\
\hline PDN $\leq 7.5$ mg/die (N/\%) & 9/36 & & \\
\hline At least one DMARD ( $\mathrm{N} / \%)$ & $13 / 52$ & & \\
\hline- MTX (N/\%) & $9 / 69.2$ & & \\
\hline$-\mathrm{HCQ}(\mathrm{N} / \%)$ & $4 / 30.7$ & & \\
\hline$-\mathrm{SSZ}(\mathrm{N} / \%)$ & $2 / 15.4$ & & \\
\hline$-\operatorname{LEF}(\mathrm{N} / \%)$ & $2 / 15.4$ & & \\
\hline I line TCZ (N/\%) & $3 / 12$ & & \\
\hline II line TCZ (N/\%) & $3 / 12$ & & \\
\hline III line TCZ (N/\%) & $9 / 36$ & & \\
\hline IV line TCZ (N/\%) & $7 / 28$ & & \\
\hline V line TCZ ( $/ \%)$ & $3 / 12$ & & \\
\hline
\end{tabular}

No difference in $\mathrm{C} 3$ and $\mathrm{C} 4$ resulted between ACPA positive and ACPA negative patients during the follow up. ESR, CRP and DAS28 levels were significanlty reduced at T1, T3, T6, T9, and T12 with the respect to T0 (Figure 1A-C). C3 levels were significantly reduced at $\mathrm{T} 3, \mathrm{~T} 6$, and $\mathrm{T} 9$ with the respect to $\mathrm{TO}$ while C4 levels were significantly reduced at $\mathrm{T} 3, \mathrm{~T} 6, \mathrm{~T} 9$, and $\mathrm{T} 12$ with the respect to T0 (Figure 1D-E). Positive correlations resulted between DAS28 and both C3 and C4 at T1 and T3 (Figure 1F-I). C3 and C4 were directly related with ESR at T1 (C3: P 0.002; C4: P 0.005), T3 (C3: P 0.04; C4: P 0.03), and T6 (C3: P<0.0001; C4: $P$ 0.002) and with CRP at T1 (C3: P 0.03; C4: P 0.01) and T6 (C3: P<0.0001; C4: $P$ 0.03). When stratifying patients in accordance with the EULAR response to therapy, $\mathrm{C} 3$ decreased significantly in responders than in no-responders at T1; moreover, responders showed lower levels of both $\mathrm{C} 3$ and $\mathrm{C} 4$ at $\mathrm{T} 1$ and $\mathrm{T} 3$ with the respect to T0 (Figure 1L-O).

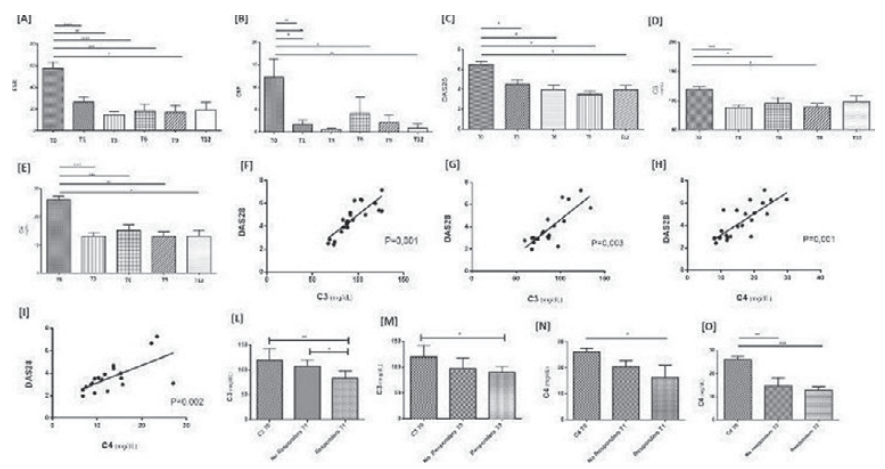

Conclusions: In RA patients, treatment with TCZ is able to reduce $\mathrm{C} 3$ and $\mathrm{C} 4$ levels in an early and substained way. The disease activity is directly related with the reduction of $\mathrm{C} 3$ and $\mathrm{C} 4$ serum levels. RA patients with a good/moderate clinical response show significantly lower $\mathrm{C} 3$ and $\mathrm{C} 4$ levels than no-responder patients. Our preliminary data suggest that $\mathrm{C} 3$ and $\mathrm{C} 4$ can be a reliable marker of both the disease activity and the response to therapy in RA patients treated with TCZ.

Disclosure of Interest: None declared

DOI: 10.1136/annrheumdis-2017-eular.4643

\section{AB0411 COMPARISON OF TUBERCULIN SKIN TEST AND INTERFERON-GAMMA RELEASE ASSAY SCREENING IN PATIENTS WITH RHEUMATOID ARTHRITIS STARTING ANTI-TUMOR NECROSIS FACTOR THERAPY}

D.T. Archimandriti ${ }^{1}$, C. lliou ${ }^{1}$, A.T. Tzallas ${ }^{2}$, E. Pelechas ${ }^{1}$, A.A. Drosos ${ }^{1}$, P.V. Voulgari ${ }^{1} .{ }^{1}$ Rheumatology Clinic, Department of Internal Medicine, Medical School, University of loannina; ${ }^{2}$ Department of Computer Engineering, Technological Educational Institute of Epirus, Greece, School of Applied Technology, Ioannina, Greece

Background: Biologic agents provide clinical benefits in patients with Rheumatoid Arthritis (RA) but they are associated with an increased risk of tuberculosis (TB) infection.

Objectives: We investigated the accordance between the tuberculin skin test (TST) and the interferon-gamma (IFN- $\gamma$ ) release assay (IGRA) for the diagnosis of latent tuberculosis infection (LTBI) in patients with RA. The objective of our study was a direct comparison between the IGRA and the TST regarding LTBI diagnosis in RA patients before starting anti-TNF agents and at least one year after the initiation of treatment.

Methods: RA consecutive patients have been included in the study both before and after 1 year of the initiation of TNF- $\alpha$ antagonist therapy. A standard questionnaire was completed for each patient, including basic demographic data and concurrent immunosuppressive therapy (corticosteroids, disease-modifying anti-rheumatic drugs [DMARDs], synthetic and biologics). The patients were screened for LTBI using IGRA and TST methods.

All analyses were performed with IBM-SPSS Statistics for Windows (version 21) The kappa coefficient $(\kappa)$ was calculated to determine the concordance between the two tests: TST and IGRA. The strength of the agreement was considered "poor" for $\kappa \leq 0.20$, "low-moderate" for $0.20<\kappa \leq 0.40$, moderate for $0.40<\kappa$ $\leq 0.60$, "substantial" for $0.60<\kappa \leq 0.80$, and "optimal" for $0.80<\kappa \leq 1$.

Results: 78 patients (67 female, 11 male) have been screened with TST and IGRA. The accordance between TST and IGRA was $89.7 \%(\kappa 0.514, \kappa<0.001)$. $A \kappa$ value of 0.514 represents a moderate accordance between the two tests. 6 female patients converted TST ( 4 became positive, and 2 became negative). No patient developed active tuberculosis.

Conclusions: A moderate accordance between the two methods (TST and IGRA) was obtained. In the absence of a diagnostic gold standard for LTBI, different issues should be taken into consideration regarding the two methods (cost, laboratory expertise and equipment etc). More studies are needed in the field in order to elucidate the cost-effectiveness but also the appropriateness of both methods.

Disclosure of Interest: None declared

DOI: 10.1136/annrheumdis-2017-eular.6400

\section{AB0412 LESS THAN 5\% OF REAL-LIFE PATIENTS WHO SWITCH FROM IV TO SC ABATACEPT IN REAL-WORLD CLINICAL PRACTICE SUBSEQUENTLY SWITCH BACK TO THE IV FORMULATION}

R. Alten $^{1}$, H.-M. Lorenz ${ }^{2}$, X. Mariette ${ }^{3}$, H. Nüßlein ${ }^{4}$, M. Galeazzi ${ }^{5}$, F. Navarro ${ }^{6}$, M. Chartier ${ }^{7}$, J. Heitzmann ${ }^{8}$, C. Rauch ${ }^{9}$, M. Le Bars ${ }^{7} .{ }^{1}$ Schlosspark-Klinik University Medicine, Berlin; ${ }^{2}$ University Hospital, Heidelberg, Germany;

${ }^{3}$ Université Paris-Sud, Paris, France: ${ }^{4}$ University of Erlangen-Nuremberg, Nuremberg, Germany; ${ }^{5}$ University of Siena, Siena, Italy; ${ }^{6}$ Hospital Univesitario Virgen Macarena, Seville, Spain; ${ }^{7}$ Bristol-Myers Squibb, Rueil-Malmaison; ${ }^{8}$ Excelya, Boulogne-Billancourt, France; ${ }^{9}$ Bristol-Myers Squibb, Munich, Germany

Background: Patients (pts) with RA may be able to switch from IV to SC abatacept with no loss of efficacy or safety concerns, but data are inconclusive. ${ }^{1-4}$ In the ACTION (AbataCepTIn rOutiNe clinical practice; NCT02109666) study, a 1-year interim analysis showed that switching had no adverse clinical effect. ${ }^{5}$ Objectives: To examine treatment patterns and explore abatacept formulation switching over 2 years in ACTION.

Methods: ACTION is a 2-year, prospective, observational study of pts with moderate-to-severe RA who initiated IV abatacept in Europe and Canada between May 2008 and December 2013. Assessments in biologic-naïve and biologic-failure pts were: baseline characteristics, rates of and reasons for switching (IV to SC), and re-switching to IV over 2 years. Descriptive data were generated: mean (SD) for continuous variables and $n(\%)$ for categorical variables. Rates of switching were estimated by Kaplan-Meier analysis. Cohorts were pooled to analyse further pts who switched owing to low numbers.

Results: In the ACTION cohort, $2350 / 2364$ pts (99.4\%) were evaluable for this analysis (673 [28.6\%] biologic naïve, 1677 [71.4\%] biologic failure). A total of 729 